Mathematical Modelling and Analysis

Volume 22 Number 2, March 2017, 167-185

https://doi.org/10.3846/13926292.2017.1285362

(c) Vilnius Gediminas Technical University, 2017
Publisher: Taylor\&Francis and VGTU

http://www.tandfonline.com/TMMA

ISSN: $1392-6292$

eISSN: 1648-3510

\title{
Mixed Fourier-Legendre Spectral Methods for the Multiple Solutions of the Schrödinger Equation on the Unit Disk
}

\section{Zhao-Xiang $\mathrm{Li}^{a, b, c}, \mathrm{Ji} \mathbf{L a o}^{b}$ and Zhong-Qing Wang ${ }^{d}$}

${ }^{a}$ Department of Mathematics, Shanghai University

Shanghai 200444, China

${ }^{b}$ Department of Mathematics, Shanghai Normal University

200234 Shanghai, China

${ }^{c}$ Scientific Computing Key Laboratory of Shanghai University

200234 Shanghai, China

${ }^{d}$ College of Science, University of Shanghai for Science and Technology 200093 Shanghai, China

E-mail(corresp.): zqwang@usst.edu.cn

E-mail: zxli@shnu.edu.cn

E-mail: 1000359505@smail.shnu.edu.cn

Received June 1, 2016; revised January 15, 2017; published online March 1, 2017

\begin{abstract}
In this paper, we first compute the multiple non-trivial solutions of the Schrödinger equation on a unit disk, by using the Liapunov-Schmidt reduction and symmetry-breaking bifurcation theory, combined with the mixed Fourier-Legendre spectral and pseudospectral methods. After that, we propose the extended systems, which can detect the symmetry-breaking bifurcation points on the branch of the $O(2)$ symmetric positive solutions. We also compute the multiple positive solutions with various symmetries of the Schrödinger equation by the branch switching method based on the Liapunov-Schmidt reduction. Finally, the bifurcation diagrams are constructed, showing the symmetry/peak breaking phenomena of the Schrödinger equation. Numerical results demonstrate the effectiveness of these approaches.
\end{abstract}

Keywords: approximation algorithm, bifurcation diagrams, multiple solutions, computational experiment, positive solution.

AMS Subject Classification: 35Q55, 35J25, 37M20, 65N35. 


\section{Introduction}

In this paper, we shall find the multiple solutions to the following nonlinear Schrödinger equation (NLS):

$$
\left\{\begin{array}{l}
F(u(x), \lambda, l):=-\Delta u(x)+\lambda u(x)+\kappa|x|^{l}|u(x)|^{p-1} u(x)=0, \quad x \in \Omega, \\
\left.u\right|_{\partial \Omega}=0
\end{array}\right.
$$

where $\Omega$ is a unit disk and $p>1, \lambda, \kappa, l$ are prescribed parameters. Its variational functional is

$$
J(u):=\int_{\Omega}\left[\frac{1}{2}\left(|\nabla u(x)|^{2}+\lambda u^{2}(x)\right)+\frac{\kappa}{p+1}|x|^{l}|u(x)|^{p+1}\right] d x .
$$

Clearly, the solutions of (1.1) correspond to the critical points of $J(u)$ in (1.2).

The $0<\mu_{1}<\mu_{2}<\cdots$ are the eigenvalues of $-\Delta$ satisfying homogeneous Dirichlet boundary condition and $\left\{v_{1}, v_{2}, \cdots\right\}$ are the corresponding eigenfunctions. The system (1.1) is called focusing (M-type) if $\kappa<0$ and $-\mu_{k+1}<\lambda<-\mu_{k}$ and defocusing (W-type) if $\kappa>0$ and $\mu_{k}<-\lambda<\mu_{k+1}$, see [25]. The previous two cases are very different in physical nature and mathematical structure. For both types, 0 is the only k-saddle. All non-trivial saddles have index $>k(<k)$ for M-type (W-type). In particular, for the Mtype with $\lambda>-\lambda_{1}, J$ is said to have a mountain pass structure and 0 is the only local minimum; for the $\mathrm{W}$-type with $k \geq 1, J$ has two local minima. In the literature, these two cases have to be treated by two very different types of variational methods.

The critical point theory has been applied to prove the existence and multiplicity of solutions of the equation (1.1) under various assumptions, see [1], [18]. However, due to the multiplicity, degeneracy and instability of the critical points with high Morse index, the computation of multiple solutions encounters essential difficulties. During the past few decades, a remarkable amount of progress has been made in the approximation approaches for the multiple solutions of some relevant problems. In the existing works, one usually computes the multiple solutions using the mountain-pass algorithm, the scaling iterative algorithm, the monotone iteration, the direct iteration algorithm, or the research and extension method, cf. [4], [8], [5], [9], [15], [24], [28], [17]. Unfortunately, the previous algorithms usually need a good guess of solution, which seems to be a difficult task.

To overcome this disadvantage, we shall use the bifurcation method [16], [27], [29] to compute the multiple solutions of (1.1). According to the bifurcation theory [11], [26], the equation (1.1) possesses nontrivial solutions, which can be bifurcated from the bifurcation points. Thereby, we can find the multiple solutions of (1.1) by using the continuation method. In actual computations, the mixed Fourier-Legendre spectral and pseudospectral methods will be employed to compute the multiple solutions.

Theoretically, we can compute the multiple solutions of the equation (1.1) for any Morse index by using the finite difference method. However, with the increase of the Morse index, the solution will oscillate rapidly. Particularly, the 
peak of the solution will occur at the boundaries as the Morse index of solutions becomes large. In this case, it is very hard to accurately simulate the behaviors of the solutions. Usually, one needs to refine the mesh, but the computational complexity is quite staggering. To do this, in this paper, we shall develop the mixed Fourier-Legendre spectral and pseudospectral methods for computing the multiple solutions cf. [19], which possess higher order accuracy than that of the finite difference method.

The multiple solutions of the NLS have been considered by some researchers. For instance, Chang et al. [3], [13] and Chen et al. [6], [22] studied the bifurcation scenario of the NLS and the multilevel spectral-Galerkin continuation methods for parameter-dependent problems. A general approach via continuation and multiple solution algorithms were given in [14].

The existence and symmetry properties of multi-bump solutions of the NLS were described in [2], [23].

The paper is organized as follows. In Section 2, we consider the multiple solutions of the Schrödinger equation (1.1) on a unit disk. We describe an algorithm and establish the mixed Fourier-Legendre spectral and pseudospectral scheme. In Section 3, the algorithm based on the bifurcation theory is applied to compute the $O(2)$ symmetric positive solutions of the problem (1.1). We take $\lambda$ or $l$ in the Schrödinger equation as a bifurcation parameter and propose the extended systems, which can detect the $O(2)-\Sigma_{1}, O(2)-\Sigma_{d}, O(2)-D_{3}$, $O(2)-D_{4}, O(2)-D_{5}, O(2)-D_{6}, O(2)-D_{7}$ and $O(2)-D_{8}$ symmetrybreaking bifurcation points on the branch of the $O(2)$ symmetric positive solutions. The $\Sigma_{1}\left(\Sigma_{d}, D_{3}, D_{4}, D_{5}, D_{6}, D_{7}, D_{8}\right)$ symmetric positive solutions are computed by the branch switching method based on the Liapunov-Schmidt reduction. Some numerical results are presented for the multiple positive solutions of the Schrödinger equation (1.1). The bifurcation diagrams are constructed, showing the symmetry/peak breaking phenomena of the Schrödinger equation. The final section is for some concluding discussions.

\section{The multiple solutions of the Schrödinger equation (1.1) on a unit disk}

\subsection{The equivariance property of (1.1)}

In this subsection, we shall discuss the symmetry properties of the nonlinear bifurcation problem (1.1) on a unit disk $\Omega$. Under the polar coordinate, the problem (1.1) may be transformed to

$$
\left\{\begin{array}{l}
F(u(r, \theta), \lambda, l):=-\partial_{r}^{2} u-\frac{1}{r} \partial_{r} u-\frac{1}{r^{2}} \partial_{\theta}^{2} u+\lambda u+\kappa r^{l}|u|^{p-1} u=0 \\
u(1, \theta)=0, \quad(r, \theta) \in[0,1] \times[0,2 \pi]
\end{array}\right.
$$

The symmetry groups of a disc are $O(2)=\left\{\mathcal{I}, S, R_{\alpha}\right\}$, where $\mathcal{I}$ is identical transformation, $S$ is reflection transformation, $R_{\alpha}$ is rotation transformation, $\alpha \in[0,2 \pi)$ is rotation angle and $\Gamma=O(2) \times Z_{2}$,

$\mathcal{I} u(r, \theta)=u(r, \theta), S u(r, \theta)=u(r,-\theta), R_{\alpha} u(r, \theta)=u(r, \theta+\alpha), Z_{2}=\{\mathcal{I},-\mathcal{I}\}$ 
The problem (2.1) is $\Gamma$ equivariant, namely

$$
F(\gamma u, \lambda, l)=\gamma F(u, \lambda, l), \quad \forall \gamma \in \Gamma
$$

and for any $\lambda \in \mathbb{R}, u \equiv 0$ is a trivial solution of the problem (2.1) with $\Gamma$ symmetry.

\subsection{Algorithm description}

In this subsection, we resolve the nontrivial multiple solutions of the Schrödinger equation (1.1) on a disc by using the Liapunov-Schmidt reduction and the numerical continuation method. Our process includes the following four steps:

(i) Calculate numerically the eigenvalue $\lambda_{0}$ and the corresponding eigenfunction $\psi_{0}$ of the operator $-\Delta$ on a unit disc, by using the mixed FourierLegendre spectral method suggested in the next subsection. Clearly, $\lambda_{0}$ and $\psi_{0}$ are the solutions of the following linearized problem:

$$
\left\{\begin{array}{l}
-\partial_{r}^{2} \psi-\frac{1}{r} \partial_{r} \psi-\frac{1}{r^{2}} \partial_{\theta}^{2} \psi=\lambda \psi, \quad(r, \theta) \in[0,1] \times[0,2 \pi], \\
\left.\psi\right|_{r=1}=0 .
\end{array}\right.
$$

(ii) Use the Liapunov-Schmidt reduction principle (cf. [10], [7]) to obtain the nontrivial solution branches near the bifurcation points. More precisely, let $u=\tau \psi_{0}+w, \eta=\lambda-\lambda_{0}$, where $w$ is an unknown function satisfying the orthogonal relationship

$$
\int_{0}^{2 \pi} \int_{0}^{1} \psi_{0}(r, \theta) w(r, \theta) r d r d \theta=0
$$

and $\tau>0$ is a small parameter. For a given small parameter $\tau$, we first resolve numerically the following system of $w$ and $\eta$, by using the mixed Fourier-Legendre pseudospectral method suggested in the next subsection and a Newton-Raphson iteration process with the initial data $\eta=0$ and $w=0$ :

$$
\begin{gathered}
-\partial_{r}^{2} w-\frac{1}{r} \partial_{r} w-\frac{1}{r^{2}} \partial_{\theta}^{2} w+\left(\eta+\lambda_{0}\right) w+\eta \tau \psi_{0}+\kappa r^{l} \mid \tau \psi_{0} \\
+\left.w\right|^{p-1}\left(\tau \psi_{0}+w\right)=0 \\
\left.w\right|_{r=1}=0, \quad \int_{0}^{2 \pi} \int_{0}^{1} \psi_{0}(r, \theta) w(r, \theta) r d r d \theta=0 .
\end{gathered}
$$

Let $\eta_{1}$ and $w_{1}$ be the approximation solutions of $\eta$ and $w$ in (2.3). Then, increasing $\tau$ gradually and resolving numerically the unknowns $\eta$ and $w$ in (2.3) by using the same process with the iterative initial data $\eta_{1}$ and $w_{1}$, we obtain new approximation solutions of $\eta$ and $w$. Repeat this process until the approximation solution of $u$ is far away from the trivial solution.

(iii) Denote by $\tau_{\text {end }}, \eta_{\text {end }}$ and $w_{\text {end }}$ the final approximation solutions of $\tau, \eta$ and $w$ in the previous process. Let $u_{\text {end }}=\tau_{\text {end }} \psi_{0}+w_{\text {end }}, \lambda_{\text {end }}=\lambda_{0}+\eta_{\text {end }}$. For a given initial data $\lambda=\lambda_{\text {end }}$, we then solve the equation (2.1) of $u$ numerically, using the suggested algorithm in the next subsection and a Newton-Raphson iteration process with the initial data $u=u_{\text {end }}$. 
(iv) Let $u_{1}$ be the approximation solution of $u$ in the previous process. Then, decreasing $\lambda$ gradually and resolving numerically the unknown $u$ in (2.1) by using the same process with the iterative initial data $u_{1}$, we obtain a new approximation solution of $u$. Repeat this process until $\lambda$ is equal to the value $\lambda$ given in (2.1).

\subsection{Mixed Fourier-Legendre spectral and pseudospectral schemes}

In this subsection, we shall construct the mixed Fourier-Legendre spectral and pseudospectral schemes (cf. [21], [20], [12]) for solving the equations (2.2), (2.3) and (2.1).

\subsubsection{Preliminaries}

Let $\left\{L_{n}(x)\right\}_{n \geq 0}, 0 \leq x \leq 1$ be the standard Legendre polynomials. They obey the orthogonality relationship:

$$
\int_{0}^{1} L_{m}(x) L_{n}(x) d x=\frac{1}{2 n+1} \delta_{m n},
$$

where $\delta_{m n}$ is the Kronecker function. The first few terms are

$$
L_{0}(x)=1, \quad L_{1}(x)=2 x-1, \quad L_{2}(x)=6 x^{2}-6 x+1 .
$$

We denote by $\left\{x_{j}, \omega_{j}\right\}_{j=0}^{N}$ the nodes of the Legendre-Gauss interpolation on the interval $(0,1)$ and the corresponding Christoffel numbers. Let $\theta_{k}=\frac{2 \pi k}{2 M+1}$, $k=0,1, \cdots, 2 M$, and $\langle u, v\rangle$ and $\langle u, v\rangle_{M, N}$ be the inner product and the discrete inner product of space $L^{2}(\Omega)$, respectively,

$$
\begin{aligned}
& \langle u, v\rangle=\int_{0}^{2 \pi} \int_{0}^{1} u(r, \theta) v(r, \theta) r d r d \theta \\
& \langle u, v\rangle_{M, N}=\frac{2 \pi}{2 M+1} \sum_{k=0}^{2 M} \sum_{j=0}^{N} u\left(x_{j}, \theta_{k}\right) v\left(x_{j}, \theta_{k}\right) x_{j} \omega_{j} .
\end{aligned}
$$

Denote

$$
\begin{gathered}
\phi_{k}(r)=c_{k}\left(L_{k}(r)-L_{k+2}(r)\right), c_{k}=1 / \sqrt{4 k+6}, \chi_{j}(r)=L_{j}(r)-L_{j+1}(r), \\
X_{M, N}=\operatorname{span}\left\{\left\{\sin (k \theta) \phi_{j}(r), \cos (k \theta) \phi_{j}(r)\right\}_{1 \leq k \leq M, 0 \leq j \leq N-2},\right. \\
\left.\left\{\chi_{j}(r)\right\}_{0 \leq j \leq N-1}\right\} .
\end{gathered}
$$

Clearly, $\chi_{j}(1)=0, \phi_{j}(0)=\phi_{j}(1)=0$. In particular, for any $\phi \in X_{M, N}$, taking $\phi(r, \theta)=\sin (m \theta) \phi_{n}(r), \cos (m \theta) \phi_{n}(r)$ and $\chi_{l}(r)$, respectively, with $m=$ $1, \cdots, M, n=0, \cdots, N-2$ and $l=0, \cdots, N-1$, we have $\partial_{\theta} \phi(0, \theta)=0$. That 
is, all functions belonging to $X_{M, N}$ are imposed the pole condition. Next, let

$$
\begin{aligned}
& a_{i j}=\int_{0}^{1} r \phi_{j}^{\prime} \phi_{i}^{\prime} d r, \quad A=\left(a_{i j}\right)_{0 \leq i, j \leq N-2}, \quad b_{i j}=\int_{0}^{1} \frac{1}{r} \phi_{j} \phi_{i} d r, \\
& B=\left(b_{i j}\right)_{0 \leq i, j \leq N-2}, \quad c_{i j}=\int_{0}^{1} r \phi_{j} \phi_{i} d r, \quad C=\left(c_{i j}\right)_{0 \leq i, j \leq N-2}, \\
& d_{i j}=\int_{0}^{1} r \chi_{j}^{\prime} \chi_{i}^{\prime} d r, \quad D=\left(d_{i j}\right)_{0 \leq i, j \leq N-1}, \\
& e_{i j}=\int_{0}^{1} r \chi_{j} \chi_{i} d r, \quad E=\left(e_{i j}\right)_{0 \leq i, j \leq N-1} .
\end{aligned}
$$

According to [19], the matrices $A$ and $B$ are symmetric tri-diagonal with

$$
a_{j k}=\left\{\begin{array}{ll}
2 j+4, & k=j+1, \\
4 j+6, & k=j,
\end{array} \quad b_{j k}= \begin{cases}-\frac{2}{j+2}, & k=j+1 \\
\frac{2(2 j+3)}{(j+1)(j+2)}, & k=j .\end{cases}\right.
$$

The matrix $\mathrm{C}$ is symmetric seven-diagonal with

$$
c_{j k}= \begin{cases}-\frac{(j+3)}{2(2 j+5)(2 j+7)}, & k=j+3, \\ -\frac{1}{4 j+10}, & k=j+2, \\ \frac{1}{2(2 j+1)(2 j+5)}+\frac{(j+3)}{2(2 j+5)(2 j+7)}, & k=j+1, \\ \frac{1}{4 j+2}+\frac{1}{4 j+10}, & k=j .\end{cases}
$$

The matrix $D$ is diagonal with $d_{i i}=2 i+2$. The matrix $E$ is symmetric penta-diagonal with

$$
e_{j k}= \begin{cases}-\frac{(j+2)}{2(2 j+3)(2 j+5)}, & k=j+2, \\ -\frac{1}{(2 j+1)(2 j+3)(2 j+5)}, & k=j+1 \\ \frac{(j+1)}{(2 j+1)(2 j+3)}, & k=j .\end{cases}
$$

\subsubsection{The mixed Fourier-Legendre spectral scheme for (2.2)}

The aim of this subsection is to compute numerically the eigenvalue $\lambda_{0}$ and the eigenfunction $\psi_{0}$ of the problem (2.2). To do this, we construct the mixed Fourier-Legendre spectral scheme. Let $a_{\lambda}(u, v)$ be the bilinear operator defined as follows:

$a_{\lambda}(u, v):=\int_{0}^{2 \pi} \int_{0}^{1} r \partial_{r} u \partial_{r} v d r d \theta+\int_{0}^{2 \pi} \int_{0}^{1} \frac{1}{r} \partial_{\theta} u \partial_{\theta} v d r d \theta-\lambda \int_{0}^{2 \pi} \int_{0}^{1} r u v d r d \theta$.

The mixed spectral approximation for (2.2) is

$$
\left\{\begin{array}{l}
\text { Find } \psi_{M, N} \in X_{M, N} \quad \text { such that } \\
a_{\lambda}\left(\psi_{M, N}, \phi\right)=0, \quad \forall \phi \in X_{M, N}
\end{array}\right.
$$

Denote

$$
\psi_{M, N}(r, \theta)=\sum_{k=1}^{M} \sum_{j=0}^{N-2}\left(\alpha_{k j} \sin (k \theta) \phi_{j}(r)+\beta_{k j} \cos (k \theta) \phi_{j}(r)\right)+\sum_{j=0}^{N-1} \gamma_{j} \chi_{j}(r),
$$




$$
\begin{aligned}
& \xi=\left(\alpha_{10}, \cdots, \alpha_{1(N-2)}, \cdots, \alpha_{M 0}, \cdots, \alpha_{M(N-2)},\right. \\
& \left.\quad \beta_{10}, \cdots, \beta_{1(N-2)}, \cdots, \beta_{M 0}, \cdots, \beta_{M(N-2)}, \gamma_{1}, \cdots, \gamma_{N-1}\right)^{\mathrm{T}} .
\end{aligned}
$$

The (2.4) is equivalent to the following linear system:

$$
\mathbb{A} \xi+\lambda \mathbb{B} \xi=0
$$

with $\mathbb{A}$ and $\mathbb{B}$ being $(2 M N-2 M+N)$ by $(2 M N-2 M+N)$ matrices. By using some existing algorithms (we here call the eig function in Matlab), we obtain numerically the eigenvalues $\lambda$ and eigenfunctions $\psi_{M, N}$ of (2.2).

\subsubsection{The mixed Fourier-Legendre pseudospectral scheme for (2.3)}

We next construct the mixed Fourier-Legendre pseudospectral scheme for (2.3). It is to find $w_{M, N} \in X_{M, N}$ and $\eta \in \mathbb{R}$ such that for any $\phi \in X_{M, N}$,

$$
\left\{\begin{array}{l}
\int_{0}^{2 \pi} \int_{0}^{1} r \partial_{r} w_{M, N} \partial_{r} \phi d r d \theta+\int_{0}^{2 \pi} \int_{0}^{1} \frac{1}{r} \partial_{\theta} w_{M, N} \partial_{\theta} \phi d r d \theta \\
+\left(\eta+\lambda_{0}\right) \int_{0}^{2 \pi} \int_{0}^{1} r w_{M, N} \phi d r d \theta+\eta \tau\left\langle\psi_{0}, \phi\right\rangle_{M, N} \\
+\kappa\left\langle r^{l}\left|\tau \psi_{0}+w_{M, N}\right|^{p-1}\left(\tau \psi_{0}+w_{M, N}\right), \phi\right\rangle_{M, N}=0, \\
\left\langle\psi_{0}, w_{M, N}\right\rangle_{M, N}=0 .
\end{array}\right.
$$

Set

$$
\begin{gathered}
w_{M, N}(r, \theta)=\sum_{k=1}^{M} \sum_{j=0}^{N-2}\left(\alpha_{k j} \sin (k \theta) \phi_{j}(r)+\beta_{k j} \cos (k \theta) \phi_{j}(r)\right)+\sum_{j=0}^{N-1} \gamma_{j} \chi_{j}(r), \\
\zeta=\left(\alpha_{10}, \cdots, \alpha_{1(N-2)}, \cdots, \alpha_{M 0}, \cdots, \alpha_{M(N-2)},\right. \\
\left.\beta_{10}, \cdots, \beta_{1(N-2)}, \cdots, \beta_{M 0}, \cdots, \beta_{M(N-2)}, \gamma_{1}, \cdots, \gamma_{N-1}\right)^{\mathrm{T}} .
\end{gathered}
$$

We find that (2.5) is equivalent to a nonlinear system, which can be formally written as:

$$
\mathbb{F}(\zeta, \eta)=0 .
$$

In actual computations, a Newton-Raphson iterative method is employed to solve the unknowns $\zeta$ and $\eta$ of the equation (2.7) numerically. Finally, we use (2.6) to obtain the approximation solution $w_{M, N}$. We can deal with the equation (2.1) by a similar process.

\subsection{Numerical results}

In this subsection, we shall compute and visualize the multiple solutions of the Schrödinger equation (1.1), by using the mixed Fourier-Legendre spectral and pseudospectral methods. 


\subsubsection{Accuracy test of algorithm}

To examine the efficiencies of our algorithm, we consider the following equation:

$$
\left\{\begin{array}{l}
-\partial_{r}^{2} u-\frac{1}{r} \partial_{r} u-\frac{1}{r^{2}} \partial_{\theta}^{2} u-u^{3}=f, \quad(r, \theta) \in[0,1] \times[0,2 \pi] \\
\left.u\right|_{r=1}=0 .
\end{array}\right.
$$

For $f \neq 0$, the equation (2.8) admits a unique solution.

Next, let $\delta u$ be the point-wise numerical error. In the following, we compare the numerical errors of our algorithm with the five points difference scheme. Take the exact solution $u(r, \theta)=(r-1) \sin (\theta)$. In Figure 1, we plot the numerical errors of the mixed Fourier-Legendre spectral method and the five points difference method with grid size $h=0.01$. We find that the numerical results of our method are much more accurate than those of the five points difference method.

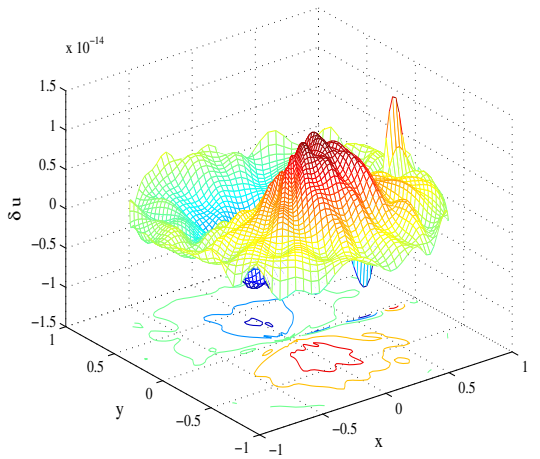

(a) The spectral method

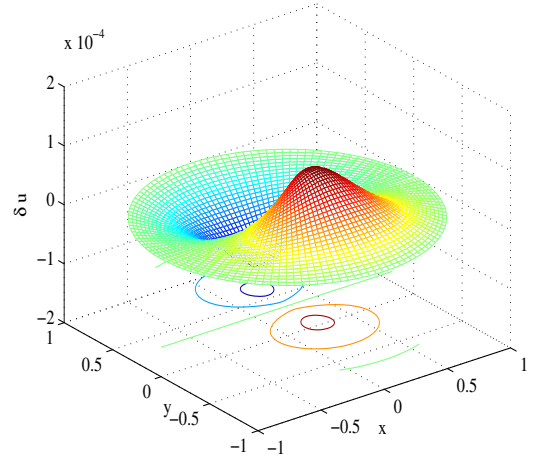

(b) The five points difference method

Figure 1. The numerical errors.

\subsubsection{The multiple solutions of (1.1)}

Let $R$ se be the residual error of (1.1), $\mathrm{J}$ is the energy as defined in (1.2). In the numerical experiments, we take $M=40, N=20$. However, in order to clearly see the mesh grids in figures, a coarse mesh is used to redraw the profiles. In Figures 2, we plot the solutions of (1.1) with $\lambda=1, p=3, \kappa=-1, l=3$ and various $\lambda_{0}$ and $\psi_{0}$. In Figures 3 , we also plot the solutions of (1.1) with $\lambda=-100, p=3, \kappa=1, l=3$ and various $\lambda_{0}$ and $\psi_{0}$. Numerical results show that it is feasible to solve the multiple solution of the nonlinear equation (1.1) by the bifurcation method. Moreover, we also find that it is not difficult to choose the initial iteration value for obtaining the multiple solutions. 


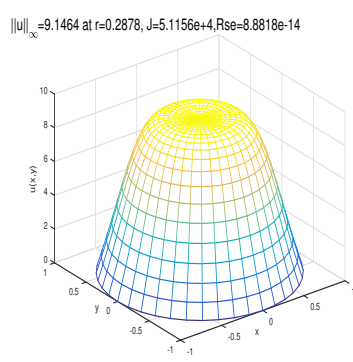

(a)

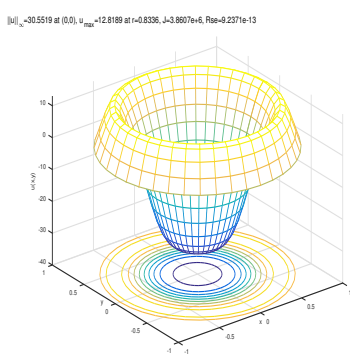

(d)

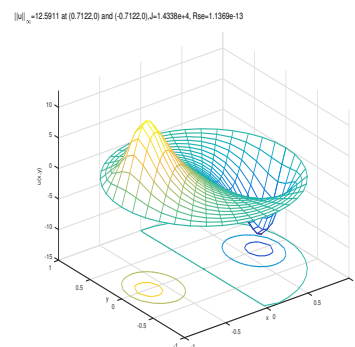

(b)

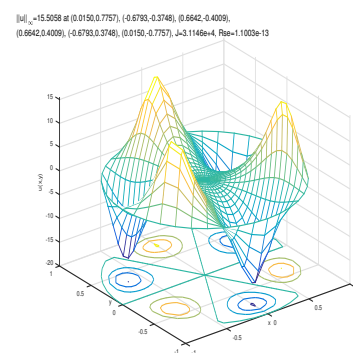

(e)

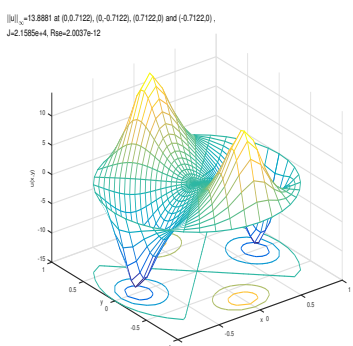

(c)

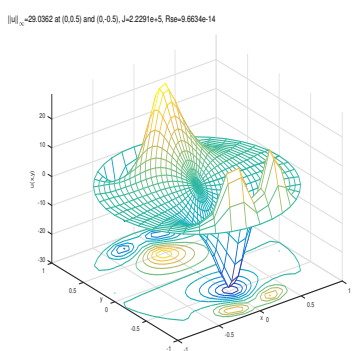

(f)

Figure 2. (a) $\lambda_{0}=5.78449, O(2)$ symmetry; (b) $\lambda_{0}=14.67342, \Sigma_{1}$ symmetry; (c) $\lambda_{0}=26.33997, \Sigma_{d}$ symmetry; (d) $\lambda_{0}=30.46207, O(2)$ symmetry; (e) $\lambda_{0}=40.59751, D_{3}$ symmetry; (f) $\lambda_{0}=57.31138, \Sigma_{1}$ symmetry.

\section{The multiple positive solutions of the Schrödinger equation (1.1) on a unit disk}

\subsection{The $O(2)$ symmetric positive solution branch}

\subsubsection{The $O(2)$ symmetric positive solution branch by $\lambda$ continua- tion}

Taking $\lambda$ as a parameter, $r$ as a constant, and using the $O(2)$ symmetric positive solutions to problem (1.1) with $\lambda=-4$ as a starting point, we get a whole $O(2)$ symmetric positive solution branch $\Gamma$ by the $\lambda$ continuation and the Newton iteration method. While $\lambda$ is continued, the eigenvalues of the Jacobian $F_{u}(u, \lambda, l)$ are monitored. We find that the eigenvalues corresponding to small absolute value are near $-2.22,2.11,7.12,12.38,17.86,23.80,30.41$ and 37.80. The corresponding eigenvectors possess $\Sigma_{1}, \Sigma_{d}, D_{3}, D_{4}, D_{5}, D_{6}, D_{7}$ and $D_{8}$ symmetry, respectively. They are the potential symmetry-breaking bifurcation points [11], [26]. Figure 4 (a) shows the whole $O(2)$ symmetric solution branch to problem (1.1) by using $\lambda$ continuation. 


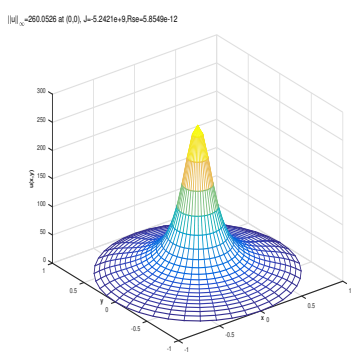

(a)

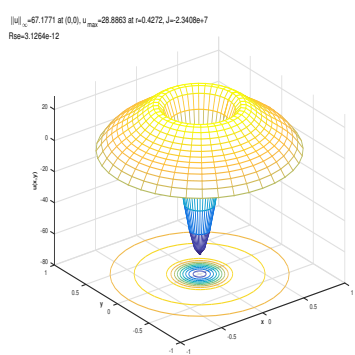

(d)

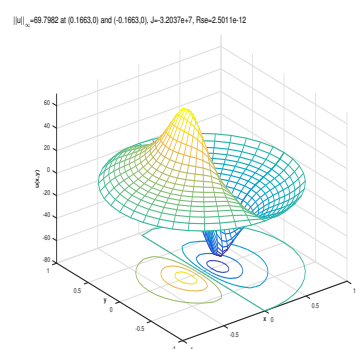

(b)

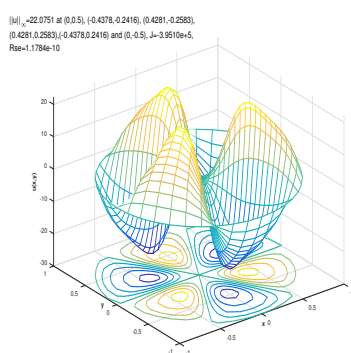

(e)

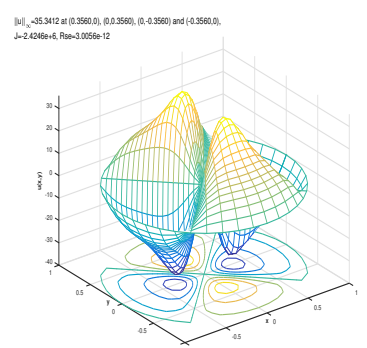

(c)

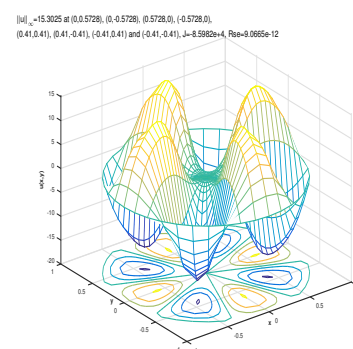

(f)

Figure 3. (a) $\lambda_{0}=5.78449, O(2)$ symmetry; (b) $\lambda_{0}=14.67342, \Sigma_{1}$ symmetry; (c) $\lambda_{0}=26.33997, \Sigma_{d}$ symmetry; (d) $\lambda_{0}=30.46207, O(2)$ symmetry; (e) $\lambda_{0}=40.59751, D_{3}$ symmetry; (f) $\lambda_{0}=57.31138, D_{4}$ symmetry.

\subsubsection{The $O(2)$ symmetric positive solution branch by $l$ continuation}

Taking $l$ as a parameter, $\lambda$ as a constant, and using the $O(2)$ symmetric positive solutions to problem (1.1) with $l=0$ as a starting point, we get a whole $O(2)$ symmetric positive solution branch $\Gamma$ by the $l$ continuation and the Newton iteration method. While $l$ is continued, the eigenvalues of the Jacobian $F_{u}(u, \lambda, l)$ are monitored. We find that the eigenvalues corresponding to small absolute value are near $0.38,2.52,4.76,7.10,9.52,11.97,14.47$ and 16.99. The corresponding eigenvectors possess $\Sigma_{1}, \Sigma_{d}, D_{3}, D_{4}, D_{5}, D_{6}, D_{7}$ and $D_{8}$ symmetry, respectively. They are the potential symmetry-breaking bifurcation points [11], [26]. Figure 6 (a) shows the whole $O(2)$ symmetric solution branch to problem (1.1) by using $l$ continuation.

\subsection{The symmetry-breaking bifurcation point on the $O(2)$ symmet- ric positive solution branch}

\subsubsection{The symmetry-breaking bifurcation point for $\lambda$ continuation}

In the following, we take $\lambda$ as a parameter and $l$ as a constant. Let $\Sigma$ be one of $\Sigma_{1}, \Sigma_{d}, D_{3}, D_{4}, D_{5}, D_{6}, D_{7}$ and $D_{8}$, and $X^{\Sigma}$ be the invariant subspace 
of $\Sigma$. Since $F(\gamma u, \lambda, l)=\gamma F(u, \lambda, l), \forall \gamma \in O(2), X^{\Sigma}$ can be decomposed into $X^{\Sigma}=X^{O(2)} \oplus W$, where $X^{O(2)}$ is the invariant subspace of $O(2), W=$ $X^{\Sigma} \cap\left(X^{O(2)}\right)^{\perp}$ and $\left(X^{O(2)}\right)^{\perp}$ is an orthogonal complement of $X^{O(2)}$. On the $O(2)$ symmetric positive solution branch, there exists a point $\left(u_{0}, \lambda_{0}\right)$, at which $F_{u}^{0}=F_{u}\left(u_{0}, \lambda_{0}, l\right)$ is singular, its null space is $\mathcal{N}\left(F_{u}^{0}\right)=\operatorname{span}\left\{\phi_{0}\right\}$ and its range space is $R\left(F_{u}^{0}\right)=\left\{x \in X^{\Sigma} \mid\left\langle\psi_{0}, x\right\rangle=0\right\}$, where $\phi_{0} \in W$ and $\psi_{0} \in W$ are null eigenvector of $F_{u}^{0}$ and $\left(F_{u}^{0}\right)^{\mathrm{T}}$, respectively. If

$$
\left\langle\psi_{0},\left(F_{u u}^{0} v_{\lambda}+F_{u \lambda}^{0}\right) \phi_{0}\right\rangle \neq 0
$$

and $v_{\lambda} \in X^{O(2)}$ is the unique solution to

$$
F_{u}^{0} v_{\lambda}+F_{\lambda}^{0}=0
$$

then the point $\left(u_{0}, \lambda_{0}\right)$ is called $O(2)-\Sigma$ symmetry-breaking bifurcation point with respect to $\lambda$.

The following is the extended system for detecting the $O(2)-\Sigma$ symmetrybreaking bifurcation point:

$$
G(y)=\left(\begin{array}{c}
F(u, \lambda, l) \\
F_{u}(u, \lambda, l) \phi \\
\left\langle h_{0}, \phi\right\rangle-1
\end{array}\right)=0,
$$

where $y=(u, \phi, \lambda)^{\mathrm{T}} \in Y=X^{O(2)} \times W \times \mathbb{R}, y_{0}=\left(u_{0}, \phi_{0}, \lambda_{0}\right)$, and $h_{0} \in W$ is a normalization of $\phi_{0}$.

Theorem 1. The extended system (3.3) is regular at the $O(2)-\Sigma$ symmetrybreaking bifurcation point $y_{0}=\left(u_{0}, \phi_{0}, \lambda_{0}\right)$.

Proof. Obviously,

$$
G_{y}^{0}=\left(\begin{array}{ccc}
F_{u}^{0} & 0 & F_{\lambda}^{0} \\
F_{u u}^{0} \phi_{0} & F_{u}^{0} & F_{u \lambda}^{0} \phi_{0} \\
0 & \left\langle h_{0}, \cdot\right\rangle & 0
\end{array}\right) .
$$

Next, we prove that $G_{y}^{0}: Y \longrightarrow Y$ is one-to-one. To this end, let

$$
G_{y}^{0} Z=0
$$

where $Z=(v, w, \alpha)^{\mathrm{T}}, v \in X^{O(2)}, w \in W, \alpha \in \mathbb{R}$. Expanding (3.4) yields

$$
\begin{aligned}
& F_{u}^{0} v+\alpha F_{\lambda}^{0}=0, \\
& F_{u u}^{0} \phi_{0} v+F_{u}^{0} w+\alpha F_{u \lambda}^{0} \phi_{0}=0, \\
& \left\langle h_{0}, w\right\rangle=0 .
\end{aligned}
$$

From (3.5), we get $v=\alpha v_{\lambda}$. Substituting $v=\alpha v_{\lambda}$ into (3.6) and taking the inner product with $\psi_{0}$ lead to $\alpha\left\langle\psi_{0},\left(F_{u u}^{0} v_{\lambda} \phi_{0}+F_{u \lambda}^{0} \phi_{0}\right)\right\rangle=0$. Therefore, by 
(3.1) we know $\alpha=0$. Further, by (3.6) we obtain $F_{u}^{0} w=0$. This, along with $\left\langle h_{0}, w\right\rangle=0$ gives that $w=0$. Similarly, we can prove that $G_{y}^{0}: Y \longrightarrow Y$ is onto. Therefore $G_{y}^{0}$ is regular.

Since $G_{y}^{0}$ is regular, we can use the mixed Fourier-Legendre pseudospectral method to discretize the equations (3.3). During the continuation, we can find certain $u^{*} \in X^{O(2)}$ and $\lambda^{*} \in \mathbb{R}$, at which the Jacobian $F_{u}\left(u^{*}, \lambda^{*}, l\right)$ has eigenvalues with small absolute value, and hence $\left(u^{*}, \lambda^{*}\right)$ and the corresponding eigenvector can be used as the initial guess for the Newton iteration. The numerical results are presented in Table 1.

Table 1. Symmetry-breaking bifurcation point for $\lambda$ continuation with $p=3, \kappa=-1$ and $l=2$.

\begin{tabular}{llllllll}
\hline & $O(2)-\Sigma_{1}$ & $O(2)-\Sigma_{d}$ & $O(2)-D_{3}$ & $O(2)-D_{4}$ & $O(2)-D_{5}$ & $O(2)-D_{6}$ & $O(2)-D_{7}$ \\
\hline$\lambda$ & -2.2231 & 2.1139 & 7.1194 & 12.3792 & 17.8551 & 23.7953 & 30.4131 \\
\hline
\end{tabular}

\subsubsection{The symmetry-breaking bifurcation point for $l$ continuation}

Taking $l$ as a parameter and $\lambda$ as a constant, we get the symmetry-breaking bifurcation point with respect to $l$. By using the similar process as in Subsection 3.2.1, we obtain the following numerical results (see, Table 2):

Table 2. Symmetry-breaking bifurcation point for $l$ continuation with $p=3, \kappa=-1$ and $\lambda=1$.

\begin{tabular}{llllllll}
\hline & $O(2)-\Sigma_{1}$ & $O(2)-\Sigma_{d}$ & $O(2)-D_{3}$ & $O(2)-D_{4}$ & $O(2)-D_{5}$ & $O(2)-D_{6}$ & $O(2)-D_{7}$ \\
\hline$l$ & 0.3785 & 2.5172 & 4.7566 & 7.0997 & 9.5136 & 11.9749 & 14.4698 \\
\hline
\end{tabular}

\subsection{Branch switching to $\Sigma$ symmetric solutions}

\subsubsection{Branch switching to $\Sigma$ symmetric solutions for $\lambda$ continuation}

We take $\lambda$ as a parameter and $l$ as a constant. Let $\left(u_{0}, \lambda_{0}\right)$ be the $O(2)-\Sigma$ symmetry-breaking bifurcation point with $u_{0} \in X^{O(2)}$. The numerical computation shows

$$
\begin{aligned}
a & :=\left\langle\psi_{0}, F_{u u}^{0} \phi_{0} \phi_{0}\right\rangle_{M, N}=0, \quad b:=\left\langle\psi_{0},\left(F_{u u}^{0} v_{\lambda}+F_{u \lambda}^{0}\right) \phi_{0}\right\rangle_{M, N} \neq 0, \\
c & :=\left\langle\psi_{0},\left(F_{u u}^{0} v_{\lambda} v_{\lambda}+2 F_{u \lambda}^{0} v_{\lambda}+F_{\lambda \lambda}^{0}\right)\right\rangle_{M, N}=0
\end{aligned}
$$

where $v_{\lambda} \in X^{O(2)}$ is the unique solution to the equation (3.2). From the algebraic bifurcation theory [11], [26], we know that at the symmetry-breaking bifurcation point, the tangent vector along the $O(2)$ symmetric positive solution branch is $\left(v_{\lambda}, 1\right)$, the tangent vector along the $\Sigma$ symmetric positive solution 
branch is $\left(\phi_{0}, 0\right)$. Next, let us define

$$
\begin{aligned}
& G(w, \eta, \varepsilon)= \begin{cases}\frac{1}{\varepsilon} F\left(u_{s}\left(\lambda_{0}+\eta\right)+\varepsilon\left(\phi_{0}+w\right), \lambda_{0}+\eta, l\right), & \varepsilon \neq 0, \\
F_{u}\left(u_{s}\left(\lambda_{0}+\eta\right), \lambda_{0}+\eta, l\right)\left(\phi_{0}+w\right), & \varepsilon=0,\end{cases} \\
& N(w, \eta, \varepsilon)=\left\langle\phi_{0}, w\right\rangle
\end{aligned}
$$

where $u_{s}\left(\lambda_{0}+\eta\right)$ is the $O(2)$ symmetric positive solutions, $w \in W$ and $\eta, \varepsilon \in \mathbb{R}$. Obviously, $G(0,0,0)=0, N(0,0,0)=0$. The Jacobian of (3.8) and (3.9) with respect to $w$ and $\eta$ at $(w, \eta, \varepsilon)=(0,0,0)$ is that

$$
A^{0}=\left.\frac{\partial(G, N)}{\partial(w, \eta)}\right|_{(0,0,0)}=\left(\begin{array}{cc}
F_{u}^{0} & B^{0} \\
\left\langle\phi_{0}, \cdot\right\rangle & 0
\end{array}\right),
$$

where

$$
B^{0}=\left[F_{u u}^{0}\left(u_{s}\left(\lambda_{0}\right), \lambda_{0}, l\right) u_{s}^{\prime}\left(\lambda_{0}\right)+F_{u \lambda}^{0}\left(u_{s}\left(\lambda_{0}\right), \lambda_{0}, l\right)\right] \phi_{0}=\left[F_{u u}^{0} v_{\lambda}+F_{u \lambda}^{0}\right] \phi_{0} .
$$

Since $\left\langle\psi_{0}, B^{0}\right\rangle=b \neq 0$ and $\phi_{0} \in \mathcal{N}\left(F_{u}^{0}\right)$, we have $B^{0} \notin R\left(F_{u}^{0}\right)$ and $\mathcal{N}\left(F_{u}^{0}\right) \cap$ $\mathcal{N}\left(\left\langle\phi_{0}, \cdot\right\rangle\right)=\{0\}$. By the Keller lemma [11], [26], $A^{0}$ is nonsingular. The implicit function theorem ensures that

$$
\left\{\begin{array}{l}
G(w, \eta, \varepsilon)=0 \\
N(w, \eta, \varepsilon)=0
\end{array}\right.
$$

have solutions $(w(\varepsilon), \eta(\varepsilon)), \forall|\varepsilon|<\varepsilon_{0}$, which can be solved by the Newton iteration method. Therefore, we obtain the $\Sigma$ symmetric positive solution branch $\left(u_{s}\left(\lambda_{0}+\eta(\varepsilon)\right)+\varepsilon\left(\phi_{0}+w(\varepsilon)\right), \lambda_{0}+\eta(\varepsilon), l\right)$ to problem (1.1), which is switched from the $O(2)$ symmetric positive solution branch.

Remark 1. During actual computations, we need not calculate the second derivative in $B^{0}$, since we always take $\varepsilon \neq 0$. Problem (3.10) possesses the following form $(\mathrm{p}=3)$ :

$$
\left\{\begin{array}{l}
-\partial_{r}^{2} w-\frac{1}{r} \partial_{r} w-\frac{1}{r^{2}} \partial_{\theta}^{2} w+\left(\varepsilon \beta_{2}+\eta+\lambda_{0}\right) w-\varepsilon \beta_{2} u_{0}\left(\lambda_{0}\right)+\left(\varepsilon \beta_{2}+\eta\right) \\
\quad \times\left(u_{0}\left(\lambda_{0}\right)+\varepsilon\left(\alpha_{2} \psi_{0}+\beta_{2} v_{\lambda}\right)\right)+\kappa r^{l}\left(3 u_{0}^{2}\left(\lambda_{0}\right) w+3 u_{0}\left(\lambda_{0}\right)\left(\varepsilon \left(\alpha_{2} \psi_{0}\right.\right.\right. \\
\left.\left.\left.\quad+\beta_{2} v_{\lambda}\right)+w\right)^{2}+\left(\varepsilon\left(\alpha_{2} \psi_{0}+\beta_{2} v_{\lambda}\right)+w\right)^{3}\right)=0 \\
\left.w\right|_{\partial \Omega}=0, \quad\left(\alpha_{2} \psi_{0}^{\mathrm{T}}+\beta_{2} v_{\lambda}^{\mathrm{T}}\right) w+\beta_{2} \eta=0 .
\end{array}\right.
$$

We can solve the equations (3.11) by the Newton iteration method and the mixed Fourier-Legendre pseudospectral method with initial value $\left(w^{0}, \eta^{0}\right)=$ $(0,0)$. When $\varepsilon$ is big enough, we can get another solution branch, see Figure $4(\mathrm{~b})$.

\subsubsection{Branch switching to $\Sigma$ symmetric solutions for $l$ continuation}

In the following, we take $l$ as a parameter and $\lambda$ as a constant. Let $\left(u_{0}, l_{0}\right)$ be the $O(2)-\Sigma$ symmetry-breaking bifurcation point with $u_{0} \in X^{O(2)}$. We can get some new positive solution branches by a similar process as in Subsection 3.3.1. 
Remark 2. The final settlement is the following form $(p=3)$ :

$$
\left\{\begin{array}{l}
-\partial_{r}^{2} w-\frac{1}{r} \partial_{r} w-\frac{1}{r^{2}} \partial_{\theta}^{2} w+\lambda w-\varepsilon \beta_{2} \kappa r^{l_{0}} u_{0}^{3}\left(l_{0}\right) \ln r-\kappa r^{l_{0}} u_{0}^{3}\left(l_{0}\right) \\
\quad \times\left(r^{\varepsilon \beta_{2}+\eta}-1\right)-3 \kappa r^{l_{0}} \varepsilon u_{0}^{2}\left(l_{0}\right)\left(\alpha_{2} \psi-\beta_{2} v_{l}\right)\left(r^{\varepsilon \beta_{2}+\eta}-1\right) \\
+\kappa r^{l_{0}+\varepsilon \beta_{2}+\eta}\left(3 u_{0}^{2}\left(l_{0}\right) w+3 u_{0}\left(l_{0}\right)\left(\varepsilon\left(\alpha_{2} \psi_{0}-\beta_{2} v_{l}\right)+w\right)^{2}\right. \\
\left.\quad+\left(\varepsilon\left(\alpha_{2} \psi_{0}-\beta_{2} v_{l}\right)+w\right)^{3}\right)=0, \\
\left.w\right|_{\partial \Omega}=0, \quad\left(\alpha_{2} \psi_{0}^{\mathrm{T}}+\beta_{2} v_{l}^{\mathrm{T}}\right) w+\beta_{2} \eta=0 .
\end{array}\right.
$$

The equations (3.12) can be solved by the Newton iteration method and the mixed Fourier-Legendre pseudospectral method with initial value $\left(w^{0}, \eta^{0}\right)=$ $(0,0)$. When $\varepsilon$ is big enough, we can get another solution branch, see Figure 6 (b).

\subsection{Numerical results}

In Figures 4(a) and 4(b), we show the $O(2)$ symmetric solution branch to problem (1.1) with $p=3, l=2$ and $\kappa=-1$, on which there are eight symmetrybreaking bifurcation points for $\lambda$ continuation. Particularly, we also exhibit in Figure 4(b) the symmetric positive solutions bifurcated from the symmetrybreaking bifurcation points, which are $\Sigma_{1}, \Sigma_{d}, D_{3}, D_{4}, D_{5}, D_{6}, D_{7}$ and $D_{8}$ symmetric, respectively, see lines 1-9.

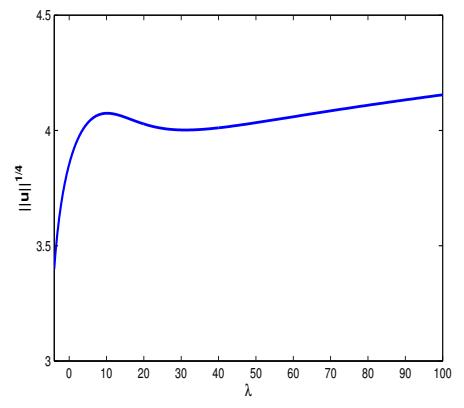

(a)

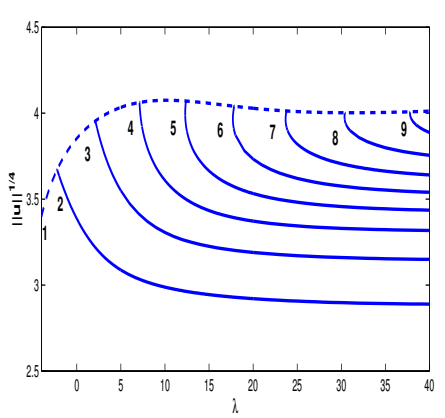

(b)

Figure 4. (a): The diagram of $O(2)$ symmetric positive solutions by $\lambda$ continuation with $\kappa=-1, p=3$ and $l=2$; (b):Symmetric positive solutions bifurcated from $O(2)$ symmetric positive solution branch with $\kappa=-1, p=3$ and $l=2$.

From Figure 4(b), we further observe that problem (1.1) has only one symmetric positive solution $(O(2))$ for $-4<\lambda<-2.2231$, two symmetric positive solutions (resp. $O(2)$ and $\Sigma_{1}$ ) for $-2.2231<\lambda<2.1139$, three symmetric positive solutions (resp. $O(2), \Sigma_{1}$ and $\Sigma_{d}$ ) for $2.1139<\lambda<7.1194$, four symmetric positive solutions (resp. $O(2), \Sigma_{1}, \Sigma_{d}$ and $D_{3}$ ) for $7.1194<\lambda<12.3792$, five symmetric positive solutions (resp. $O(2), \Sigma_{1}, \Sigma_{d}, D_{3}$ and $D_{4}$ ) for $12.3792<$ $\lambda<17.8551$, six symmetric positive solutions (resp. $O(2), \Sigma_{1}, \Sigma_{d}, D_{3}, D_{4}$ and 
$D_{5}$ ) for $17.8551<\lambda<23.7953$, seven symmetric positive solutions (resp. $O(2), \Sigma_{1}, \Sigma_{d}, D_{3}, D_{4}, D_{5}$ and $\left.D_{6}\right)$ for $23.7953<\lambda<30.4131$, eight symmetric positive solutions (resp. $O(2), \Sigma_{1}, \Sigma_{d}, D_{3}, D_{4}, D_{5}, D_{6}$ and $D_{7}$ ) for $30.4131<$ $\lambda<37.7955$, nine symmetric positive solutions (resp. $O(2), \Sigma_{1}, \Sigma_{d}, D_{3}, D_{4}, D_{5}$, $D_{6}, D_{7}$ and $\left.D_{8}\right)$ for $\lambda>37.7955$.

The previous nine symmetric positive solutions to problem (1.1) with $p=3$, $l=2$ and $\lambda=40$ are also plotted in Figure 5.

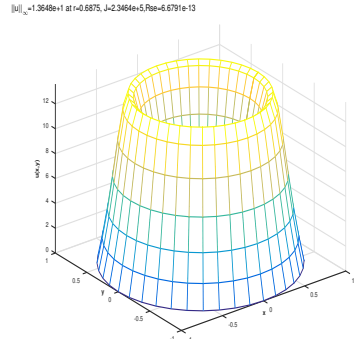

(a)

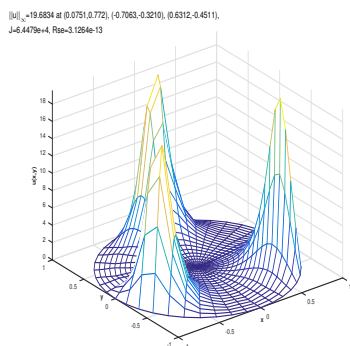

(d)

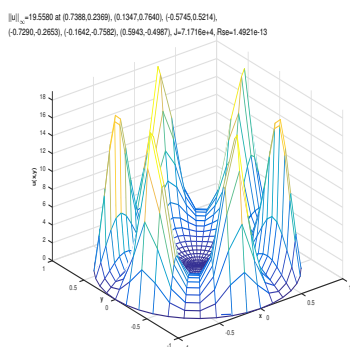

(g)

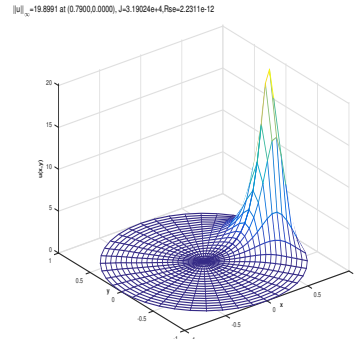

(b)

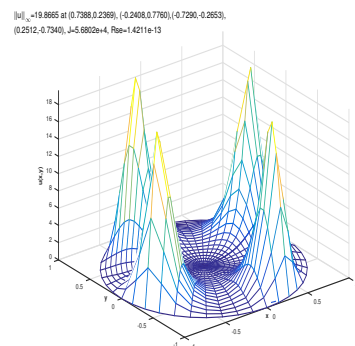

(e)

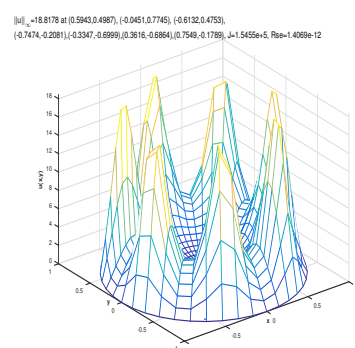

(h)

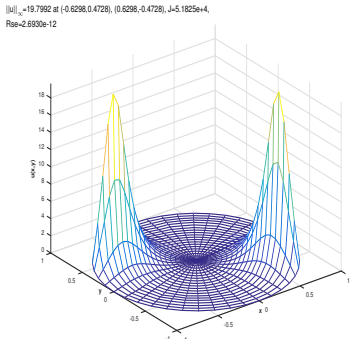

(c)

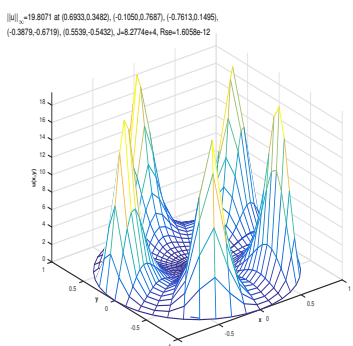

(f)

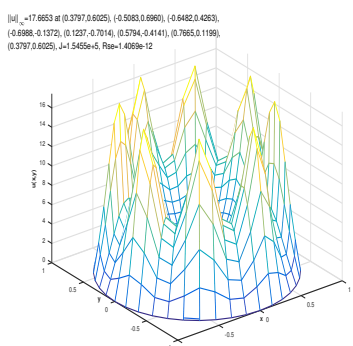

(i)

Figure 5. Nine positive solutions to problem (1.1) with $p=3, l=2, \lambda=40$ and $\kappa=-1$, which are $O(2), \Sigma_{1}, \Sigma_{d}, D_{3}, D_{4}, D_{5}, D_{6}, D_{7}$ and $D_{8}$ symmetric, respectively.

In Figures 6(a) and 6(b), we show the $O(2)$ symmetric solution branch to problem (1.1) with $p=3, \lambda=1$ and $\kappa=-1$, on which there are eight 
symmetry-breaking bifurcation points for $l$ continuation. Moreover, we also exhibit in Figure 6(b) the symmetric positive solutions bifurcated from the symmetry-breaking bifurcation points, which are $\Sigma_{1}, \Sigma_{d}, D_{3}, D_{4}, D_{5}, D_{6}$, $D_{7}$ and $D_{8}$ symmetric, respectively, see lines 1-9. From Figure 6(b), we further observe that problem (1.1) has only one symmetric positive solution $O(2)$ for $0<l<0.3785$, two symmetric positive solutions (resp. $O(2)$ and $\Sigma_{1}$ ) for $0.3785<l<2.5172$, three symmetric positive solutions (resp. $O(2), \Sigma_{1}$ and $\Sigma_{d}$ ) for $2.5172<l<4.7566$, four symmetric positive solutions (resp. $O(2), \Sigma_{1}, \Sigma_{d}$ and $\left.D_{3}\right)$ for $4.7566<l<7.0997$, five symmetric positive solutions (resp. $O(2), \Sigma_{1}, \Sigma_{d}, D_{3}$ and $D_{4}$ ) for $7.0997<l<9.5136$, six symmetric positive solutions (resp. $O(2), \Sigma_{1}, \Sigma_{d}, D_{3}, D_{4}$ and $D_{5}$ ) for $9.5136<l<$ 11.9749 , seven symmetric positive solutions (resp. $O(2), \Sigma_{1}, \Sigma_{d}, D_{3}, D_{4}, D_{5}$ and $D_{6}$ ) for $11.9749<l<14.4698$, eight symmetric positive solutions (resp. $O(2), \Sigma_{1}, \Sigma_{d}, D_{3}, D_{4}, D_{5}, D_{6}$ and $\left.D_{7}\right)$ for $14.4698<l<16.98622$, nine symmetric positive solutions (resp. $O(2), \Sigma_{1}, \Sigma_{d}, D_{3}, D_{4}, D_{5}, D_{6}, D_{7}$ and $D_{8}$ ) for $l>16.98622$.

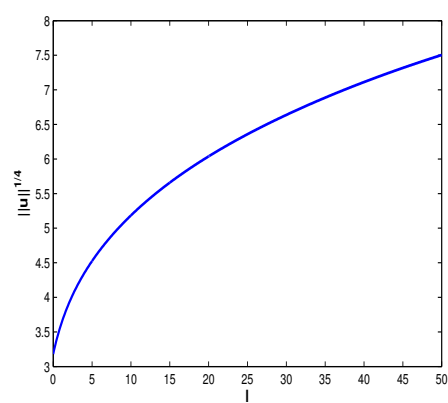

(a)

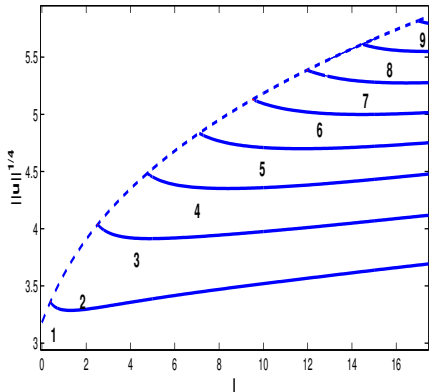

(b)

Figure 6. (a): The diagram of $O(2)$ symmetric positive solutions by $l$ continuation with $\kappa=-1, p=3$ and $\lambda=1$; (b): Symmetric positive solutions bifurcated from $O(2)$ symmetric positive solution branch with $\kappa=-1, p=3$ and $\lambda=1$.

The previous nine symmetric positive solutions to problem (1.1) with $p=3$, $l=17.5$ and $\lambda=1$ are also plotted in Figure 7 .

\section{Conclusions}

In this paper, based on the Liapunov-Schmidt reduction and symmetry-breaking bifurcation theory, we compute and visualize the multiple solutions of the Schrödinger equation on a unit disc, using the mixed Fourier-Legendre spectral and pseudospectral methods. Starting from the non-trivial solution branches of the corresponding nonlinear bifurcation problem, we obtain the multiple solutions of Schrödinger equation with various symmetries numerically. The bifurcation diagrams are constructed, showing the symmetry/peak breaking phenomena of the Schrödinger equation. Numerical results demonstrate the 


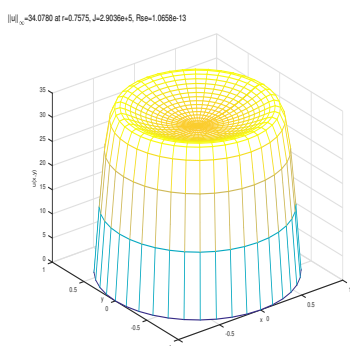

(a)

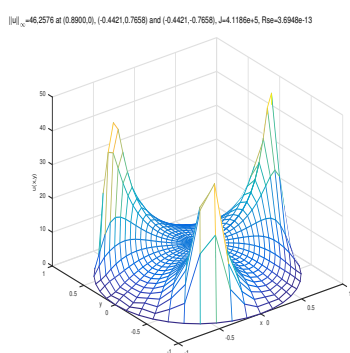

(d)

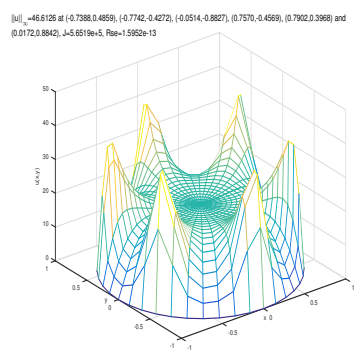

(g)

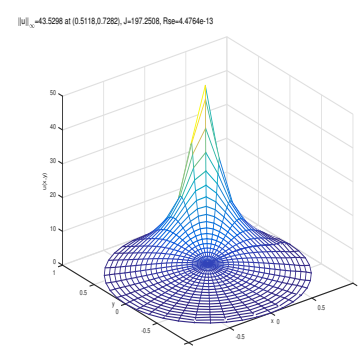

(b)

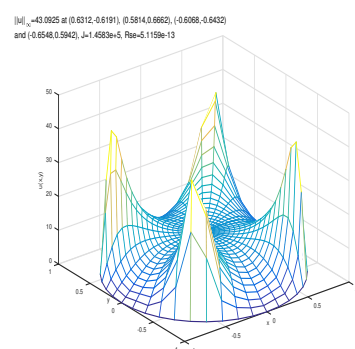

(e)

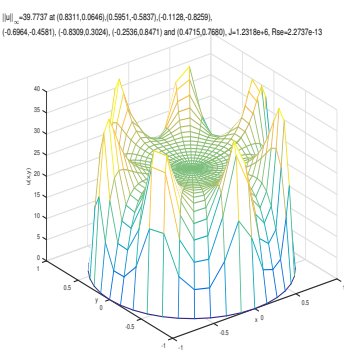

(h)

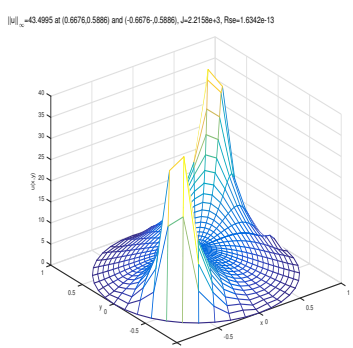

(c)

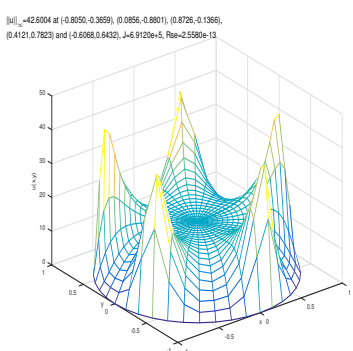

(f)

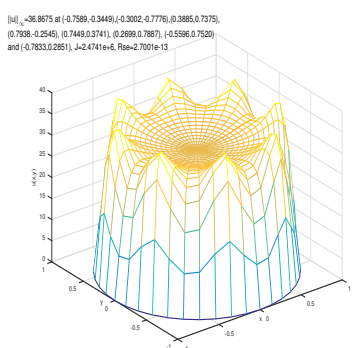

(i)

Figure 7. Nine positive solutions to problem (1.1) with $p=3, l=17.5, \lambda=1$ and $\kappa=-1$, which are $O(2), \Sigma_{1}, \Sigma_{d}, D_{3}, D_{4}, D_{5}, D_{6}, D_{7}$ and $D_{8}$ symmetric, respectively.

effectiveness of these approaches. The main merits of our method include: (i) We can compute the multiple solutions of the Schrödinger equation with a larger Morse index and various symmetries; (ii) The suggested algorithms can overcome effectively the difficulty for searching the initial guess encountered in some other popular algorithms. (iii) The proposed algorithms are a highorder accuracy method. Moreover, numerical experiments also indicate that it is much easier to find the nontrivial solutions with our method, especially the positive solutions during the computational process. 


\section{Acknowledgements}

The work of the first author was partially supported by Innovation Program of Shanghai MEC (No.14YZ078), NSF of Shanghai (No.15ZR1430900, 14ZR1431300) and China Postdoctoral Science Foundation (No.2015M571538). The work of the third author was partially supported by NSF of China (No. 11571238) and the Research Fund for Doctoral Program of Higher Education of China (No. 20133127110006). I would like to thank two anonymous referees, whose comments have helped to improve the paper.

\section{References}

[1] A. Ambrosetti and P.H. Rabinowitz. Dual variational methods in critical point theory and applications. Journal of Functional Analysis, 49(4):349-381, 1973.

[2] A.R. Champneys and B. Sandstede. Numerical Computation of Coherent Structures. 2007.

[3] S.L. Chang, C.S. Chien and B.W. Jeng. Liapunov-Schmidt reduction and continuation for nonlinear Schrödinger equations. Siam Journal on Scientific Computing, 29(2):729-755, 2007. https://doi.org/10.1137/050642861.

[4] C. Chen and Z. Xie. Search extension method for multiple solutions of a nonlinear problem . Computers 85 Mathematics with Applications, 47(2-3):327-343, 2004.

[5] G. Chen, Y. Deng, W.M. Ni and J. Zhou. Boundary element monotone iteration scheme for semilinear elliptic partial differential equations, part ii: Quasimonotone iteration for coupled systems. Mathematics of Computation of the American Mathematical Society, 65(215):943-982, 1996.

[6] H.S. Chen and C.S. Chien. Multilevel spectral-Galerkin and continuation methods for nonlinear Schrödinger equations. Siam Journal on Multiscale Modeling E Simulation, 8(2):370-392, 2009. https://doi.org/10.1137/090749177.

[7] Y. Chen and A.Y.T. Leung. Bifurcation and chaos in engineering. Springer Berlin, 1998. https://doi.org/10.1007/978-1-4471-1575-5.

[8] Y.S. Choi. A mountain pass method for the numerical solution of semilinear elliptic problems. Nonlinear Analysis, 20(4):417-437, 1993.

[9] Z. Ding, D. Costa and G. Chen. A high-linking algorithm for sign-changing solutions of semilinear elliptic equations. Nonlinear Analysis Theory Methods \& $\mathrm{Ap}$ plications, 38(2):151-172, 1999. https://doi.org/10.1016/S0362-546X(98)000868.

[10] M. Golubitsky and D.G. Schaeffer. Singularities and Groups in Bifurcation Theory Volume I. Springer New York, 1985.

[11] M. Golubitsky, I. Stewart and D. Schaeffer. Singularities and groups in bifurcation theory. Vol. II. Springer-Verlag, 1988. https://doi.org/10.1007/978-1-46125034-0.

[12] B.Y. Guo. Spectral Methods and Their Applications. World Scientific, 1998.

[13] B.W. Jeng, C.S. Chien and I.L. Chern. Spectral collocation and a two-level continuation scheme for dipolar Bose-Einstein condensates. Journal of Computational Physics, 256:713-727, 2014. https://doi.org/10.1016/j.jcp.2013.09.018. 
[14] C. Kuehn. Efficient gluing of numerical continuation and a multiple solution method for elliptic PDEs. Applied Mathematics \& Computation, 266:656-674, 2015. https://doi.org/10.1016/j.amc.2015.05.120.

[15] Y. Li and J. Zhou. A minimax method for finding multiple critical points and its applications to semilinear PDE. Siam Journal on Scientific Computing, 23(3):840-865, 2001. https://doi.org/10.1137/S1064827599365641.

[16] Z.X. Li and Z.Q. Wang. Pseudospectral methods for computing the multiple solutions of the Lane-Emden equation. Journal of Computational Physics, 255(6):407-421, 2013.

[17] C.V. Pao. Block monotone iterative methods for numerical solutions of nonlinear elliptic equations. Numerische Mathematik, 72(2):239-262, 1995. https://doi.org/10.1007/s002110050168.

[18] P. Rabinowitz. Minimax Methods in Critical Point Theory with Applications to Differential Equations. Published for the Conference Board of the Mathematical Sciences by the American Mathematical Society, 1986. https://doi.org/10.1090/cbms/065.

[19] J. Shen. Efficient spectral-Galerkin methods iii: Polar and cylindrical geometries. Siam Journal on Scientific Computing, 18(6):1583-1604, 1997. https://doi.org/10.1137/S1064827595295301.

[20] J. Shen and T. Tang. Spectral and high-order methods with applications. Science Press, 2006.

[21] J. Shen, T. Tang and L.L. Wang. Spectral methods : algorithms, analysis and applications. Springer, 2011. https://doi.org/10.1007/978-3-540-71041-7.

[22] Y.S. Wang and C.S. Chien. A two-parameter continuation method for computing numerical solutions of spin-1 Bose-Einstein condensates. Journal of Computational Physics, 256:198-213, 2014. https://doi.org/10.1016/j.jcp.2013.08.056.

[23] Z.Q. Wang. Existence and symmetry of multi-bump solutions for nonlinear Schrödinger equations. Journal of Differential Equations, 159(1):102-137, 1999. https://doi.org/10.1006/jdeq.1999.3650.

[24] Z.Q. Wang and J. Zhou. A local minimax-Newton method for finding multiple saddle points with symmetries. Siam Journal on Numerical Analysis, 42(4):1745-1759, 2004. https://doi.org/10.1137/S0036142903431675.

[25] Z. Xie, W. Yi and J. Zhou. An augmented singular transform and its partial newton method for finding new solutions. Journal of Computational \& Applied Mathematics, 286:145-157, 2015. https://doi.org/10.1016/j.cam.2015.02.049.

[26] Z.H. Yang. Non-linear Bifurcation: Theory and Computation. Science Press, 2007.

[27] Z.H. Yang, Z.X. Li and H.L. Zhu. Bifurcation method for solving multiple positive solutions to Henon equation. Science China Mathematics, 51(12):2330-2342, 2008. https://doi.org/10.1007/s11425-007-0198-x.

[28] J. Zhou. A local min-orthogonal method for finding multiple saddle points. Journal of Mathematical Analysis \& Applications, 291(1):66-81, 2002. https://doi.org/10.1016/j.jmaa.2003.10.043.

[29] H.L. Zhu, Z.X. Li and Z.H. Yang. Newton's method based on bifurcation for solving multiple solutions of nonlinear elliptic equations. Mathematical Methods in the Applied Sciences, 36(16):2208-2223, 2013. 